\title{
Paramagnetism and Reentrant Behavior in Quasi-One-Dimensional Superconductors at High Magnetic Fields
}

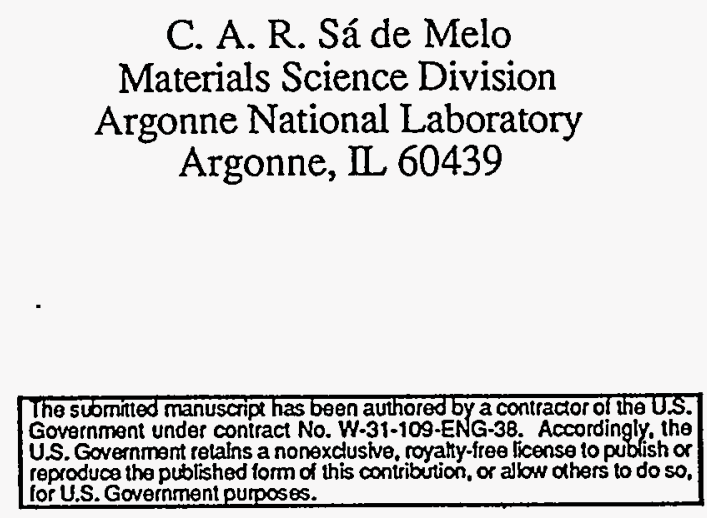

Materials Science Division

rgonne National Laboratory

Argonne, IL 60439

$/ \mathrm{sm}$

Distribution:

1-2. M. J. Masek

3. B. D. Dunlap

4. G. W. Crabtree

5. A. A. Abrikosov

6. F. Y. Fradin

7. M. B. Brodsky

8. M. R. Norman

9. Editorial Office

10. Authors

11. S\&TC (5)

This work was supported by the National Science Foundation Office of Science and Technology Center under Contract No. DMR 91-20000 and by the Division of Materials Sciences, Office of Basic Energy Sciences of DOE, under contract No. W-31-109-ENG-38.

\section{MASTER}




\title{
Paramagnetism and Reentrant Behavior in Quasi-One-Dimensional Superconductors at High Magnetic Fields
}

\author{
C. A. R. Sá de Melo \\ Materials Science Division \\ Argonne National Laboratory \\ Argonne, IL 60439
}

$/ \mathrm{sm}$

\section{DISCLAIMER}

\begin{abstract}
This report was prepared as an account of work sponsored by an agency of the United States Government. Neither the United States Government nor any agency thereof, nor any of their employees, makes any warranty, express or implied, or assumes any legal liability or responsibility for the accuracy, completeness, or usefulness of any information, apparatus, product, or process disclosed, or represents that its use would not infringe privately owned rights. Reference herein to any specific commercial product, process, or service by trade name, trademark, manufacturer, or otherwise does not necessarily constitute or imply its endorsement, recommendation, or favoring by the United States Government or any agency thereof. The views and opinions of authors expressed herein do not necessarily state or reflect those of the United States Government or any agency thereof.
\end{abstract}

This work was supported by the National Science Foundation Office of Science and Technology Center under Contract No. DMR 91-20000 and by the Division of Materials Sciences, Office of Basic Energy Sciences of DOE, under contract No. W-31-109-ENG-38. 


\title{
Paramagnetism and Reentrant Behavior in Quasi-One-Dimensional Superconductors
}

\author{
at High Magnetic Fields
}

\author{
C. A. R. Sá de Melo \\ Argonne National Laboratory, Materials Science Division, Argonne, IL 60439
}

The thermodynamics of quasi-one-dimensional superconductors in the presence . of large magnetic fields is studied. When the quantum effects of the magnetic field - are taken into account, several reentrant superconducting phases persist at very high fields. In the last reentrant phase the free energy change, the specific heat jump and the excess magnetization are estimated near the critical temperature. In particular; the excess magnetization is found to be paramagnetic as opposed to diamagnetic in weak fields and its sign is controlled by the slope of $H_{c_{2}}(T)$. We further generalize this result to the entire phase diagram (including all quantum phases) and to different physical systems using general thermodynamic relations which show that the sign of the excess magnetization $\Delta M$ of the superconducting state near $H_{c_{2}}(T)$ follows $d H_{c_{2}}(T) / d T$. These relations provide a scenario for the evolution of the sign of $\Delta M$ from weak fields to strong fields.

PACS numbers : $74.20-\mathrm{z}, 74.70 . \mathrm{Kn}, 74.90 .+\mathrm{n}$

Typeset Using REVTEX 
In recent years quasi-onc-dimensional (quasi-1D) conductors with open fermi surfaces have received a lot of attention[1] because of their unusual properties in the presence of a magnetic field. The most representative conductors of this class are the Bechgaard salts which exhibit Field Induced Spin Density Waves (FISDW) phases at high magnetic fields due to a quantized nesting mechanism[1,2]. These organic salts have a very rich phase diagram and are also known to be superconducting. It is then very natural to ask what will be the effect of large magnetic fields in systems that are superconducting at zero magnetic field.

High magnetic fields usually tend to destroy superconductivity because of orbital frustration effects that raise the free energy and suppress the coherence of the superconducting state. This picture usually emerges in the framework of a GinzburgLandau theory, where the semiclassical approximation is used and the quantum effects of the magnetic field are completely neglected. For instance, in systems with closed Fermi surfaces, such as isotropic superconductors, the effects of Landau level quantization in the normal state have to be taken into account in order to determine the transition temperature $T_{c}(H)$ for large magnetic fields[3], i.e., when the cyclotron frequency $\omega_{c} \gg 2 \pi T$. On the other hand, in systems such as quasi-1D superconductors, where the Fermi surface is open, i.e, has two disconnected branches, there is no Landau level quantization effects in the normal state, since the semiclassical electronic orbits are also open. Lebed[4] was the first to propose that quasi-1D superconductors, for equal spin triplet pairing, may exhibit a strong reentrant phase in high magnetic fields.

More recently Dupuis, Montambaux and Sá de Melo[5] have shown that the phase diagram originally proposed by Lebed[4] was incomplete.. Provided that the system is in the clean limit, for both singlet and triplet superconductors, many additional intermediate reentrant phases exist between the semiclassical and the extreme quantum 
regimes. They are separated by first order transition lines corresponding to struct.ural phase transitions between laminar lattices with different periods. The laminar lattices are characterized by two indices, $Q$ and $M ; Q$ is a modulation wavevector along the direction of largest hopping ( $x$ direction) and $M$ is the periodicity of the laminar lattice along the direction of smallest hopping ( $z$ direction). The periodicity $M$ is commensurate with the underlying crystalline lattice.

In this paper we will be concerned mostly with laminar lattices of rectangular symmetry. Moreover, we will mainly discuss the high field (extreme quantum limit, $M=1, Q=0)$ phase[5] of the equal spin triplet superconductors, where many analytical approximations can be made to calculate the free energy, the change in specific heat and the excess magnetization close to the critical temperature $T_{c}(H)$. In particular, we show that the excess magnetization in the last reentrant phase is paramagnetic due to localization and quantum effects as opposed to diamagnetic in .weak.fields. We further generalize this result to all quantum phases by means of general thermodynamic relations which indicate that the sign excess magnetization. $\Delta M$ of the superconducting state near $H_{c_{2}}(T)$ follows $d H_{c_{2}}(T) / d T$. In addition, these relations provide a scenario for the evolution of the sign of $\Delta M$ from weak fields to strong fields.

. To understand the properties and the nature of the superconducting state of quasiID superconductors in the presence of magnetic fields it is important to understand first the normal state. We chose the branch dispersion $\varepsilon_{\alpha, \sigma}(\mathrm{k})=v_{F}\left(\alpha k_{x}-k_{F}\right)+$ $t_{y} \cos \left(k_{y} b\right)+t_{z} \cos \left(k_{z} c\right)-\sigma \mu_{B} H$ and take advantage of the largest $\left(E_{F}\right)$ and smallest $\left(t_{z}\right)$ scales, $E_{F} \gg t_{y} \gg t_{z}$, in the problem at zero field by applying the magnetic field along the $y$ direction $(\mathrm{H}=H \hat{y})$. We will concentrate here only on the quantum aspects of the problem. Using the Landau gauge $\mathrm{A}=(H z, 0,0)$ where $k_{x}, k_{y}$ and $\alpha$ are good quantum numbers, while $k_{z}$ is not, the eigenfunctions of $H_{0}(\mathrm{k}-e \mathrm{~A})=$ 
$E_{\alpha, \sigma}(\mathrm{k})+\alpha \omega_{\mathrm{c}} z / c$ are $\Phi_{k_{x}, k_{y}, N, \alpha, \sigma}(x, y, z=n c)=\exp \left\{i\left(k_{x} x+k_{y} y\right)\right] J_{N^{\prime}-n}\left(\alpha t_{z} / \omega_{c}\right)$ and the eigenvalues $\epsilon_{k_{x}, k_{y}, N, \alpha, \sigma}=\varepsilon_{\alpha, \sigma}\left(\mathrm{k}_{p}\right)+\alpha N \omega_{\mathrm{c}}$ where $J_{p}(u)$ is the Bessel function of integer order $p$ and argument $u$ and $\varepsilon_{\alpha, \sigma}\left(\mathrm{k}_{p}\right)=v_{F}\left(\alpha k_{x}-k_{F}\right)+t_{y} \cos \left(k_{y} b\right)-\sigma \mu_{B} H$ is a 2D dispersion [6], with $\omega_{c}=v_{F} G$ and $G=|e| H c . G$ is the characteristic magnetic wavenumber and $\omega_{c}$ is the frequency of the periodic motion along the $z$ direction (analogous to the cyclotron frequency for isotropic systems), i.e., the frequency at which the electrons traverse the Brilouin zone in the $z$ direction. Notice that the eigenfunctions for the quasi-1D case become localized when $\omega_{c} \gg t_{z}$ in the planes $z=N c$, i.e., $x y$ plane, via the argument of the Bessel function in agreement with the semiclassical condition that the amplitude of motion in the $z$ direction $z_{A} \ll c$. [4] [5] The physical interpretation is even more transparent when we analyse in addition the energetics; the energy barrier for an electron to tunnel from plane $z=n c$ to plane $z=(n+1) c$ is $\omega_{c}$, hence when the energy barrier becomes large in comparison to the liopping $t_{z}$, the electrons are essentially confined to their initial planes.

In order to study the instability of the normal state for the quasi-1D systems we add to the non-interacting Hamiltonian density in the presence of the magnetic field $\left(\hbar=k_{B}=1\right)$

$$
H_{0}(\mathbf{r})=\sum_{\alpha, \sigma} \psi_{\alpha, \sigma}^{\dagger}(\dot{\mathrm{r}}) \varepsilon_{\alpha, \sigma}(\hat{\mathrm{k}}-e \mathrm{~A}) \dot{\psi}_{\alpha, \sigma}(\mathrm{r})
$$

an interaction Hamiltonian $H_{\text {int }}=H_{S S}+H_{T S}$ involving a singlet part $H_{S S}=$ $-\lambda_{S S} \int d \mathbf{r} O_{S S}^{\dagger}(r) O_{S S}^{\dagger}(\mathbf{r})$ and $H_{T S}=-\lambda_{T S} \sum_{\gamma} \int d \mathbf{r} O_{T S, \gamma}^{\dagger}(\mathbf{r}) O_{T S, \gamma}(\mathbf{r})$, where the operators $O_{S S}(\mathbf{r})=\sum_{\sigma} \sigma \psi_{-,-\sigma}(\mathbf{r}) \psi_{+, \sigma}(\mathbf{r})$ and $O_{T S, \gamma}(\mathbf{r})=\sum_{\sigma, \sigma^{\prime}} \sigma \psi_{-,-\sigma}(\mathbf{r}) \tau_{\sigma, \sigma^{\prime}}^{(\gamma)} \psi_{+, \sigma^{\prime}}$ with $\tau_{\sigma, \sigma^{\prime}}^{(\gamma)}$ being the elements of the Pauli matrices, and the index $\gamma=1,2,3(x, y, z)$.

To study the instability of the normal state we consider here only the situation where $t_{:} \gg T_{c}^{(\eta)}(0)$, with $\eta=S S, T S$, which guarantees that there is no Josephson coupling $\left(\xi_{z}(0) \gg c\right)$ in the superconducting state between different $x y$ planes at zero 
magnetic ficld. First we analyse the upper critical ficld $H_{c_{2}}(T)$ or $T_{c}(H)$. In weak magnetic fields where both the quantum effects of the magnetic ficld $\omega_{c} \ll 2 \pi T$ and the Zccman splitting can be neglected the semiclassical approximation can be used to obtain $H_{c_{2}}(T)=\phi_{0} / 2 \pi \xi_{x}(T) \xi_{z}(T$.$) lcading to T_{c}^{(\eta)}(H)=T_{c}^{(\eta)}(0)-\kappa t_{z} \omega_{c} / T_{c}^{(\eta)}(0)$ with $\kappa=7 \sqrt{2} \zeta(3) / 16 \pi^{2}$, where $T_{c}^{(\eta)}=2 \omega_{D} \gamma / \pi \exp \left[-1 / N\left(E_{F}\right) \lambda_{\eta}\right]$ and $\omega_{D} \sim E_{F}$. In the limit of high magnetic fields $\omega_{c} \gg 2 \pi T$ the quantum effects and localization induced by the magnetic field play an important role in the determination of $T_{c}(H)$ as well as the Zeeman splitting. In the case of singlet pairing or opposite spin triplet pairing the Zeeman term strongly suppresses the transition to the superconducting state. In contrast for equal spin triplet pairing (ESTP) the Zeeman splitting does not play any important role and $T_{c}(\infty) \rightarrow T_{c}^{(2 D)}(0)$. since the elec- . tronic motion is confined to the $x y$ planes which are parallel to the direction of the applied magnetic field, i.e., the orbital frustration that destroys superconductivity . is suppressed. In the ESTP case for $\omega_{c} \gg t_{z} \gg T_{c}(H)$ the critical temperature is $T_{c}^{(T S)}(H) \simeq T_{c}^{(2 D)}(0)\left[1-\left(t_{z} / \omega_{c}\right)^{2} \ln \left[\gamma \omega_{c} / \pi T_{c}^{(2 D)}(0)\right]\right]$, a limiting result first obtained by Lebed[4].

- Now we turn our attention to the analysis of the thermodynamics of the superconducting state for the case of ESTP. Near $T_{c}(H)$ the total free energy in the static approximation $\Delta_{\nu}(\mathbf{r}, \tau)=\Delta_{\nu}(\mathbf{r})$ can then be written as

$$
\dot{F}=\dot{F_{0}}+F_{1}+F_{2}+F_{3}+F_{4}+F_{B}
$$

where

$$
F_{1}=\left(1 / \lambda_{T S}\right) \sum_{\nu} \int d \tau d \mathbf{r}\left|\Delta_{\nu}(\mathrm{r})\right|^{2}, F_{2}=\sum_{\nu_{1}, \nu_{2}} \int d \mathrm{r}_{1} d \mathrm{r}_{2} \Delta_{\nu_{1}}^{*}\left(\mathrm{r}_{1}\right) \Delta_{\nu_{2}}\left(\mathrm{r}_{2}\right) K_{2}\left(\mathrm{r}_{1}, \mathrm{r}_{2}\right) \delta_{\nu_{1}, \nu_{2}} \text {, }
$$
$F_{3}$

$$
=\quad \sum_{\nu_{1}, \nu_{2}} \int d \mathrm{r}_{1} d \mathrm{r}_{2} \Delta_{\nu_{1}}^{*}\left(\mathrm{r}_{1}\right) \Delta_{\nu_{2}}\left(\mathrm{r}_{2}\right) K_{3}\left(\mathrm{r}_{1}, \mathrm{r}_{2}\right) \delta_{\nu_{1}, \nu_{2}}
$$

and $F_{4}=\sum_{\left\{\nu_{j}\right\}} \int\left\{d \mathrm{r}_{j}\right\} \Delta_{\nu_{1}}^{*}\left(\mathrm{r}_{1}\right) \Delta_{\nu_{2}}\left(\mathrm{r}_{2}\right) \Delta_{\nu_{3}}\left(\mathrm{r}_{3}\right) \Delta_{\nu_{4}}^{*}\left(\mathrm{r}_{4}\right) K_{4}\left(\left\{\mathrm{r}_{j}\right\}\right) \tilde{\delta}$, with $\tilde{\delta}=\delta_{\nu_{1}+\nu_{4}, \nu_{2}+\nu_{3}}$ and $F_{B}=\int d \mathrm{r}[B(\mathrm{r})]^{2}$. The Kernels $K_{i}$ are expressed in terms of the nor- 
mal state Green's functions. $K_{2}$ is responsible for the instability of the normal state, $K_{3}$ generates the induced supercurrents and the fiernel $K_{4}$ determines the stability of the superconducting phase. In addition, $\nabla \wedge \nabla \wedge$ $\mathbf{a}_{s}(\mathbf{r})=4 \pi \mathbf{j}_{s}(\mathbf{r})$ with $\mathbf{j}_{s}(\mathbf{r})=\sum_{\nu} \int d \mathrm{r}_{1} \mathbf{r}_{2} \Delta_{\nu}^{*}\left(\mathbf{r}_{1}\right)\left[\delta K_{3}\left(\mathrm{r}_{1}, \mathbf{r}_{2}\right) / \delta \mathbf{a}_{s}(\mathbf{r})\right] \Delta_{\nu}\left(\mathrm{r}_{2}\right)$ and $\mathbf{A}_{T}=\mathbf{A}+\mathbf{a}_{s}$. For calculation purposes we use the gauge $\mathbf{A}=(0,0,-H x)$ where the current operator $\hat{\jmath}(\mathrm{r})$ is defined by its components, $\hat{\jmath}_{x}(\mathrm{r})=\alpha|e| v_{F}$; $\hat{\jmath}_{y}(\mathbf{r})=-|e| t_{y} b \sin \left(b \partial_{y}\right) ; \hat{\jmath}_{z}(\mathbf{r}) \doteq-|e| t_{z} c \sin \left(c \partial_{z}-i G \dot{x}\right) ;$ and the Green's function $G_{\alpha, \sigma}\left(i \omega, \mathbf{r}, \mathbf{r}^{\prime}\right)=\sum_{\left\{k_{\rho}\right\}} \exp i\left[\mathbf{k}_{\rho} \cdot \rho\right] Z_{n, n^{\prime}} g_{\alpha, \sigma}\left(i \omega, \mathbf{k}_{\rho}\right)$ with the wave function renormalization factor $Z_{n, n^{\prime}}=\sum_{N} J_{N-n}\left(\bar{\alpha} t_{z} / \omega_{c}\right) J_{N-n^{\prime}}\left(\bar{\alpha} t_{z} / \omega_{c}\right) f_{n, n^{\prime}}\left(k_{x}\right)$, where in addition $f_{n, n^{\prime}}=\exp i\left[\left(n x-n^{\prime} x^{\prime}\right) G\right]$. Here $g_{\alpha, \sigma}\left(i \omega, \mathrm{k}_{\rho}\right)=1 /\left[i \omega-\dot{\varepsilon}_{\alpha, \sigma}\left(\mathrm{k}_{\rho}\right)\right]$ is the two dimensional Green's function.

In the ESTP state we chose the non-uniform order parameter to be of the form $\Delta(\mathrm{r})=\Delta_{0}+2 \Delta_{2} \cos (2 G x)$, expected to be valid in the last reentrant phase $M=1, Q=0$, which has periodicity $l_{x}=\pi / \dot{G}$. and $l_{z}=c$ and holds a flux quantum inside the plaquette $\left(l_{x}, l_{z}\right)$, i.e., $H l_{x} l_{z}=\phi_{0}$. In the range of fields we are interested here $\omega_{c} \gg t_{z}, \Delta_{0} \gg \Delta_{2}$ and the free energies $F_{i}$ have the form, $F_{1} \simeq-\left(1 / \lambda_{T S}\right)\left(\Delta_{0}^{2}+2 \Delta_{2}^{2}\right) ;$ $F_{2} \simeq B_{20} \Delta_{0}^{2}+B_{11} \Delta_{0} \Delta_{2}+B_{02} \Delta_{2}^{2} ; \quad F_{3} \simeq D_{20} \Delta_{0}^{2}+D_{11} \Delta_{0} \Delta_{2}+D_{02} \Delta_{2}^{2} ; F_{4} \simeq$ $(1 / 2)\left(C_{40} \Delta_{0}^{4}+C_{31} \Delta_{0}^{3} \Delta_{2}+C_{22} \Delta_{0}^{2} \Delta_{2}^{2}\right)$. From the saddle point condition $\delta F / \delta \Delta_{0}=\cdot 0$, $\delta F / \delta \Delta_{2}=\dot{0}, \delta F / \delta \mathrm{a}_{s}=0$, we find $\Delta_{0}^{2} \simeq\left[8 \pi^{2} / 7 \zeta(3)\right] T_{c}(H)\left[T_{c}(H)-T\right]\left[1+\left(t_{z} / \omega_{c}\right)^{2}\right]$ and $\Delta_{2} \simeq\left(t_{z} / \omega_{c}\right)^{2} \Delta_{0} / 2$ (for $E_{F} \gg \omega_{c}$ ), leading to the order parameter $\Delta(\mathrm{r})=$ $\Delta_{0}\left[1+\left(\dot{t}_{z} / \omega_{c}\right)^{2} \cos (2 G x)\right]$, while $j_{x}(\mathrm{r}) \simeq 0 ; j_{y}(\mathrm{r})=0 ; j_{z}(\mathrm{r}) \simeq j_{0}(T, H) \sin (2 G x)$ and $j_{0}(T, H)=8|e|\left(t_{z} / \omega_{c}\right)^{2} c N\left(E_{F}\right) \ln \left[\gamma \omega_{c} / \pi T_{c}(H)\right] \Delta_{0}^{2}$, where all expressions are correct to order $\left(t_{z} / \omega_{c}\right)^{2}$. This magnetic field induced Josephson coupling $j_{0}(T, H)$ is a consequence of the localization $\left(\omega_{c} \gg t_{z}\right)$ and quantum effects $\left(\omega_{c} \gg 2 \pi T\right)$ of the magnetic field and it is absent in weak fields in the regime we have considered here $\left(t_{z} \gg T_{c}(0)\right)$. As a result the normal state in high fields becomes unstable towards a weakly coupled 
qualsi-2D superconductor with a magnetic: field induced Josephson coupling between different axy planes. This result should be contrasted to the usual Josephson coupling present, in weakly coupled layered superconductors $\left(t_{z} \ll T_{c}(0)\right)$ even in the absence of a magnetic field. [7] We should also mention here that the gauge invariant form of $j_{z}(\mathrm{r})$ involves the phase difference $\Delta \phi=\phi_{N^{+1}}(\mathrm{r})-\phi_{N}(\mathrm{r})+2|e| \int \mathrm{A} \cdot d \mathrm{~s}$, in the case we are considering here $\phi_{N+1}(\mathrm{r})=\phi_{N}(\mathrm{r})$ and the expression for $j_{z}(\mathrm{r})$ is gauge invariant as it must be.

The local magnetic induction $\mathrm{B}(\mathrm{r})=\mathrm{H}+\mathrm{h}_{s}(\mathrm{r})$ can be calculated from $\nabla \wedge \mathbf{h}_{s}(\mathbf{r})=$ $4 \pi j(r)$. The boundary conditions in calculating $h_{s}$ are very important, we consider the length of the system in the $x$ direction to be $L_{x}$, and along the planes $x=0$ and $x=L_{x}$ we impose that $\mathrm{B}(\mathrm{r})=\mathrm{H}$, i.e, $\mathbf{h}_{s}(0)=\mathbf{h}_{s}\left(L_{x}\right)=0$. The induced field $\mathbf{h}_{s}(\mathrm{r})=$ $h_{s}(x) \hat{y}$, where $h_{s}(x)=4 \pi\left[j_{0}(T, H) / 2 G\right][1-\cos (2 G x)]$ with the number of flux quanta $N_{x}$ enclosed between two consecutive $x y$ planes being $N_{x}=L_{x} G / \pi$ (this ensures. . the appropriate boundary conditions for $\left.\mathbf{h}_{\dot{s}}(\mathbf{r})\right)$. The local excess magnetization per plaquette is $\Delta \mathrm{M}(\mathrm{r})=\Delta M(\mathrm{r}) \hat{y}$, where $\Delta M(\mathrm{r})=\mathrm{h}_{s}(\mathrm{r}) / 4 \pi$. Hence the net excess magnetization per.plaquette. $\Delta M=j_{0}(T, H) / 2 G$ is paramagnetic, i.e., $\Delta M>0$, a : rather surprising result at first when contrasted with the usual diamagnetism in.weak fields.

To check the correctness of the above result (not a minus sign error), let us calculate the free energy difference $\Delta F=F_{s}-F_{n}$, between the superconducting and normal states. The saddle point $\Delta F$ is given by $\Delta F \simeq-\left[8 \pi^{2} / 7 \zeta(3)\right] N\left(E_{F}\right)\left[T-T_{c}(H)\right]^{2}+F_{b}$, where $F_{b}=\int d \mathbf{r}\left[h_{s}(\mathbf{r})\right]^{2} / 8 \pi$ where only the terms correct to order $\left(t_{z} / \omega_{c}\right)^{2}$ should be considered for consistency. The total excess magnetization can be calculated from $\Delta F$ from the thermodynamic relation $\Delta \mathrm{M}=\mathrm{B} / 4 \pi-\partial \Delta F / \partial \mathrm{B}$ which leads to the following result $\Delta \mathrm{M} \simeq\left[16 \pi^{2} / \tau \zeta(3)\right] N\left(E_{F}\right)\left[T_{c}(H)-T\right]\left[d T_{c}(H) / d H\right] \hat{y}$. We find then that the sign of the excess magnetization is controlled by the slope of $T_{c}(H)$. The 
excess magnetization calculated from the free energy and from the induced supercurrent.s are the same to the order of the approximation $\left(t_{z} / \omega_{c}\right)^{2}$ considered here, as expected. This confirms the paramagnetic behavior obtained from the supercurrents and further associates the predicted paramagnetism with the reentrant behavior in large fields, i.e., $d T_{c}(H) / d H>0$.[8] In addition, the excess entropy is negative as it must be, $\Delta S \simeq-\left[16 \pi^{2} / 7 \zeta(3)\right] N\left(E_{F}\right)\left[T_{c}(H)-T\right]$, since the superconducting state is more ordered than the normal state. A direct calculation of specific heat jump $\triangle C$ from $\Delta F$ leads to $\Delta C \simeq\left[16 \pi^{2} / 7 \zeta(3)\right] N\left(E_{F}\right) T_{c}(H)$, which deviates from the BCS expression only through $T_{c}(H)$. We would like to emphasize though that the relation between the sign of $\Delta M$ and the slope $d T_{c}(H) / d H$ or $d H_{c_{2}}(T) / d T$ is neither a result of the mean field approximation, nor a consequence of the microscopic model applied . to the specific quasi-1D system. This relation has its origins on pure thermodynamic considerations and it is quite general as we shall see next.

Now, from the general thermodynamic relations in the vicinity of a second order phase transition, the specific heat change at constant $H, \Delta C=$ : $T\left[d H_{c 2}(T) / d T\right]^{2}(\partial \Delta M / \partial H)_{T}$, as well as $(\partial \Delta S / \partial H)_{T}=-d H_{c 2}(T) / d T(\partial \Delta M / \partial H)_{T}$. Our results for $\Delta C$ and $\Delta M$ do obey these general thermodynamic relations, but most . interestingly is the relation

$$
\Delta S=-\left(\alpha_{m} / \alpha_{s}\right)\left(d H_{c_{2}}(T) / d T\right) \Delta M
$$

with $\alpha_{s}>0$ and $\alpha_{m}>0$, which shows that if $\Delta S<0$ the product $\left(d H_{c_{2}}(T) / d T\right) \Delta M>0$, i.e., a diamagnetic (paramagnetic) excess magnetization $\Delta M<0(\Delta M>0)$ is always linked to a decrease (increase) in $H_{c_{2}}(T), d H_{c_{2}} / d T<0$ $\left(d H_{c_{2}} / d T>0\right)$. Conversely, when $T_{c}(H)$ decreases (increases) with $H$ there is a diamagnetic (paramagnetic) excess magnetization. The last relation is derived from the simple observation that in a sccond order phase transition $\Delta M$ and $\triangle S$ must 
vanish at $H_{c_{2}}$ (from below), hence in the vicinity of $H_{c_{2}} \Delta M=M_{0}\left(1-H / H_{c_{2}}\right)^{a_{m}}$ and $\Delta S=S_{0}\left(1-H / H_{c_{2}}\right)^{a_{a}}$. () ur results also satisfy this relation with $\alpha_{m}=\alpha_{s}=1$, since the saddle point approximation is a mean field theory. Furthermore, the results are also valid even when fluctuation cffects around the mean field thcory are considered, provided that the system is in thermodynamic equilibrium, since they only affect equation (3) numerically, i.e., via the ratio $\alpha_{m} / \alpha_{s}(>0)$ of the critical exponents. In addition, we should emphasize that the pure thermodynamic derivation given in this paragraph does not invoke any particular microscopic or phenomenological model as an approximation to the free energy in order to establish the relation between the sign of $\triangle M$ and the slope of $T_{c}(H)$, which is valid over the entire phase diagram.

These thermodynamic relations can also be applied to any system in the vicinity of a second order phase transition and in particular to the case of the Chevrel superconductor $E u_{x} S n_{1-x} M o_{6} S_{8}[9]$, where paramagnetism and reentrant behavior have already been observed experimentally. The microscopic origin of this effect in $E u_{x} S n_{1-x} M o_{6} S_{8}$ is very distinct from the quasi-1D (ESTP) system considered here, where the reentrant behavior is a consequence of a localization effect induced by the magnetic field and a dimensional crossover from $3 D$ to $2 D$ superconductivity. The reentrant behavior here is purely orbital in nature as opposed to the case of the $E u_{x} S n_{1-x} M o_{6} S_{8}$, where spins play aṇ essential role (Jaccarino-Peter effect). Furthermore, here the quantum effects of the field $\omega_{c} \gg 2 \pi T$ also play an important role while they are not needed to explain the observed effect in $E u_{x} S n_{1-x} M o_{6} S_{8}$.[9] To make additional contact with experiments we should emphasize that the excess magnetization $\Delta M$ is the magnetization arising only from the superconducting part of the entire system. Therefore, to measure $\Delta M$, the additional contributions to the total magnetization must be remored by an extraction method as in the case of $E u_{x} S n_{1-x} M o_{6} S_{8} \cdot[9]$ Furthermore, the expected paramagnetism in the last ESTP 
phase of the quasi-1D Bechgaard salts should occur for temperatures $T<2 K^{\circ}$ and ficlds $H>5 T$, when the parameters $t_{z}=20 K, T_{c}=2 K$ are used.

We have also analysed the laminar phases $M=2, Q=G$ for the rectangular lattice, $M=2, Q=0$ for the triangular lattice. In both cases supercurrents flow along $x$ direction and the $z$ direction and the period of the Josephson vortex lattice in the $z$ direction is $2 c$. The form of the order parameter does not change within a given phase, but its magnitude and phase along the plaquette are magnetic field dependent. This magnetic field dependence is also transfered to the local supercurrents, which in turn present both paramagnetic and diamagnetic flows within a given plaquette. It is the competition between these current flows as a function of $H$ that determines the net $\Delta M$ which is positive (paramagnetic) or negative (diamagnetic) depending on the slope of $T_{c}(H)$ as if the.dominant charge carriers were Cooper pairs of holes or electrons respectively. With the aid of relation (3) we can extend this scenario to the entire phase diagram and, in particular, to all intermediate quantum phases of quasi1D superconductors. There, the sign of $\Delta M$ has an oscillatory behavior governed by $d H_{c_{2}}(T) / d H$ as the magnetic field is increased. For instance, in a given phase the sign of $\Delta M$ changes continously following the slope of $H_{c_{2}}(T)$, while the sign of $\Delta M$ changes discontinuously between consecutive phases since $H_{c_{2}}(T)$ has cusps with slopes of opposite signs. A similar scenario is also applicable to the isotropic case in high fields, where the competition between paramagnetic and diamagnetic currents in the Abrikosov lattice produces an excess $\Delta M$ whose sign follows the slope of $H_{c_{2}}(T) .[10]$

Before concluding, we must say that in the quasi-1D superconductors like Bechgaard salts, the possible reentrant intermediate phases even for the best situation (triplet superconductivity) are in the very. low temperature regime $T_{c}(H) \ll T_{c}(0)$. Eventhough these salts are very clean materials, $\left[1 / 2 \pi T_{c}(0) \tau \simeq 0.016\right][1]$, in the in- 
t.ermediate field regime the impurity effects may be very dramatic and destroy superconductivity, since $T_{c}(H)$ is strongly suppressed $\left[1 / 2 \pi T_{c}(H) \tau \gg 1\right]$. At least the last recntrant phases $M=1$ and $M=2$ are expected to survive small disorder effects. In contrast, the effect of thermal fluctuations in strong fields $t_{z} / \omega_{\mathrm{c}} \gg 1$ may be very important, thus requiring further studies of the exact nature of the transition.

We have presented a discussion of the thermodynamics of the extreme quantum limit phase of the quasi-1D superconductors close to $T_{c}(H)$, where we calculated the changes in free energy $(\Delta F)$, in specific heat $(\Delta C)$ and in magnetization $(\Delta M)$. We showed that $\Delta C$ and $\triangle M$ deviate from the weak field results when the quantum effects of the magnetic field $H$ are incorporated. In particular, $\Delta M$ is positive (paramagnetic) in the extreme quantum limit as opposed to the negative (diamagnetic) in the weak field limit and its sign is controlled by $d H_{c_{2}}(T) / d T$, i.e., $d T_{c}(H) / d H$. In addition, we have showed that general thermodynamic relations require the sign of the excess. magnetization $\Delta M$ to be controlled by the slope $d H_{c_{2}}(T) / d T$ in the vicinity of $H_{c_{2}}(T)$. We emphasized the applicability of this result to different systems as well as to the evolution of the sign of $\Delta M$ over the entire phase diagram of quasi-1D systems.

We would like to thank A. A. Abrikosov for encouragement, M. R. Norman, $\mathrm{N}$. Dupuis and G. Montambaux for the critical reading of an earlier version of the manuscript. This work was supported by the National Science Foundation Office of Science and Technology Centers under the contract No. DMR 91-20000 and by the U.S. Department of Energy, Basic Energy Sciences-Material Sciences, under the contract No. W-31-109-ENG-38. 


\section{REFERENCES}

[1] "The Physics and Chemistry of Organic Superconductors ", Edited by G. Saito and S. Kagoshima, (Springer Verlag, 1987).

[2] G. Montambaux, Physica Scripta T 35, 188 (1991).

[3] For a review and references see M. Rasolt and Z. Tesanovic, Rev. Mod. Phys. 64 709 (1992).

[4] A.:G. Lebed', JETP Lett. 44, 114 (1986); L. I. Burlachkov, L. P. Gorkov and.A. G. Lebed', Europhys. Lett. 4, 941 (1987).

[5] N. Dupuis, G. Montambaux and C. A. R. Sá de Melo, Phys. Rev. Lett. 70, 2613

[6] The quasi-1D eigenspectrum is a function of both $k_{x}$ and $k_{y}$ since only one degree of freedom $\left(k_{z}\right)$ is absorbed into it due to the periodic motion along the $z$ direction. Hence, the eigenspectrum is two-dimensional in constrast with the isotropic case.

[7] L. N. Bulaeviskii, Int. J. Mod. Phys. B 4, 1849 (1990)

[8]. A similar effect is found both experimentally and theoretically for the $T=0$ excess magnetization in the FISDW phases of quasi-1D conductors, see G. Montambaux, M. J. Naughton, R. V. Chamberlin, X. Yan, P. M. Chaikin, M. Ya. Azbel, Phys. Rev. B 39, 885 (1989).

[9] Ø. Fischer, H. W. Meul, M. G. Karkut, G. Remenyi, L. Welp; J. C. Piccoche and K. Maki, Phys. Rev. Lett. 55, 2972 (1985).

[10] M. R. Norman (private communication) 